\title{
Efecto histoprotector de Aloe vera L. "sábila" en ratas con daño gástrico provocado por indometacina
}

\author{
Cytoprotective effect of Aloe vera L. "aloe" in rats with gastric damage caused by indomethacin \\ Efeito citoprotetor de Aloe vera L. "aloe" em ratos com lesão gástrica causada por indometacina
}

\author{
Luis Gonzales-Llontop \\ luis.gonzales@untrm.edu.pe \\ https://orcid.org/0000-0001-7944-2642 \\ Universidad Nacional Toribio Rodríguez de Mendoza \\ de Amazonas, Chachapoyas, Perú
}

\author{
Mariel Chotón-Calvo \\ mariel.choton@untrm.edu.pe \\ https://orcid.org/0000-0001-6870-9268
}

Universidad Nacional Toribio Rodríguez de Mendoza de Amazonas, Chachapoyas, Perú

\author{
Julio Chico-Ruiz \\ jchico@unitru.edu.pe \\ https://orcid.org/0000-0002-7287-321X \\ Universidad Nacional de Trujillo, Trujillo, Perú
}

Artículo recibido 15 de marzo 2021 / Arbitrado y aceptado 06 de abril 2021 / Publicado 04 de mayo 2021

\section{RESUMEN}

Se evaluó el efecto histoprotector de Aloe vera L. "sábila" en ratas con daño gástrico provocado por indometacina. Sé utilizó 50 ratas, de 4 meses de edad y de $250 \mathrm{~g}$ de peso promedio. Los animales se distribuyeron en grupo de diez para cada uno de los tratamientos. El grupo 1 (testigo) recibió SSF $(0,9 \%)$, el grupo 2 (control) se administró indometacina $(20 \mathrm{mg} / \mathrm{kg})$, y los grupos problema $(3,4$ y 5$)$ recibieron una, dos y tres dosis de sábila (50 $\mathrm{mg} / \mathrm{kg}$ ), respectivamente. La primera dosis se administró a las 4 horas procedentes a la indometacina y las demás dosis con sucesiones de 4 horas. Transcurrida una hora fueron anestesiadas y sacrificadas. En los niveles de lesiones gástricas macroscópicas y microscópicas se hallaron significativamente diferencias entre el grupo control $(p<0.001)$ y los grupos problema. En los grupos tratados con $A$. vera $L$. $(p>0.05)$ no se encontró diferencias significativas. En nuestras condiciones experimentales la aplicación de un extracto de sábila en ratas con daño gástrico presentó efecto histoprotector.

Palabras clave: Aloe vera L.; indometacina; úlceras; ratas; medicina natural

\section{ABSTRACT}

The cytoprotective effect of Aloe vera $\mathrm{L}$. "aloe" was evaluated in rats with gastric damage caused by indomethacin. 50 rats, 4 months old and $250 \mathrm{~g}$ of average weight, were used. The animals were distributed in a group of ten for each of the treatments. Group 1 (control) received SSF $(0.9 \%)$, group 2 (control) received indomethacin (20 $\mathrm{mg} / \mathrm{kg})$, and problem groups (3, 4, and 5) received one, two, and three doses of aloe (50 mg / kg), respectively. The first dose was administered 4 hours before indomethacin and the other doses in 4-hour successions. After one hour they were anesthetized and euthanized. In the levels of macroscopic and microscopic gastric lesions, significant differences were found between the control group ( $p$ $<0.001$ ) and the problem groups. In the groups treated with $A$. vera $L$. ( $p>$ 0.05 ), no significant differences were found. In our experimental conditions the application of an extract of aloe in rats with gastric damage showed histoprotective effect.

Key words:Aloe vera L.; indomethacin; ulcers; rats; natural medicine

\section{RESUMO}

O efeito histoprotetor de Aloe vera L. "aloe" foi avaliado em ratos com lesão gástrica causada por indometacina. Foram utilizados 50 ratos, com 4 meses de idade e $250 \mathrm{~g}$ de peso médio. Os animais foram distribuídos em grupos de 10. O grupo 1 (controle) recebeu SSF $(0,9 \%)$, o grupo 2 (controle) recebeu indometacina $(20 \mathrm{mg} / \mathrm{kg}$ ) e os grupos problemáticos $(3,4$ e 5$)$ receberam um, dois e três doses de Aloe vera L. (50 mg / kg), respectivamente. A primeira dose foi administrada 4 horas antes da indometacina e as outras doses em sucessões de 4 horas. Após uma hora, eles foram anestesiados e sacrificados. Nos níveis de lesões gástricas macroscópicas e microscópicas, diferenças significativas foram encontradas entre o grupo controle ( $p<0,001)$ e os grupos problemáticos. Nos grupos tratados com Aloe vera L. ( $>>0,05)$ não foram encontradas diferenças significativas. Em nossas condições experimentais, a aplicação de um extrato de Aloe vera L. "aloe" em ratos com lesão gástrica apresentou efeito histoprotetor.

Palavras-chave: Aloe vera L.; indometacina; úlceras; ratos; medicina natural 


\section{INTRODUCCIÓN}

Las patologías gástricas son afecciones muy frecuentes En los diferentes estratos de la sociedad moderna. Los orígenes que motivan el inicio de una injuria gástrica no se conocen con exactitud, sin embargo se acepta a la influencia de factores como las preocupaciones, la ansiedad, la tensión emocional, los procesos infecciosos y la dieta alimenticia ejerciendo un acusado influjo tanto en la aparición de la enfermedad, como en la agudización de los síntomas (1).

Es pues la medicina alternativa $y$ complementaria una opción de un nuevo tratamiento para sofocar ciertas dolencias sin tener que recurrir a los productos químicos farmacéuticos pocos accesibles por sus costos y en algunos casos causantes de enfermedades gástricas debido a su uso excesivo o a su composición de fabricación. Se estima que miles de productos farmacológicos cada año irrumpen en el mercado; existiendo poca información sobre las reacciones adversas en el cuerpo humano que ocasionan los AINES tales como el ibuprofeno, la indometacina, ibuprofeno y otros teniendo muchos de ellos elevado consumo por receta médica y/o por su automedicación (2). Los AINES constituyen uno de los agentes terapéuticos más utilizados en la práctica médica diaria, bien como tratamiento de afecciones reumáticas o como parte del tratamiento sintomático o etiológico del dolor; no obstante, éstos presentan evidentes manifestaciones adversas a nivel gástrico, siendo la gastrolesividad, una de ellas; atribuyéndose dicho efecto, entre otros, a la disminución de la síntesis de prostaglandinas, por inhibición de la Cox uno y la Cox dos. Las poblaciones nativas utilizan las plantas, usando técnicas sencillas, dependiendo del nivel técnico del curandero, de los medios disponibles y la tradición $(3,4)$.

La indometacina junto al ibuprofeno, naproxeno $(5,6)$ ketorolaco $(7)$ yotros pertenece a la familia de los AINES, cuyo nombre químico es el ácido arilalcanoico 3-(5-Nitrofurfuciliden) amino-2-oxazolidona. Es un fármaco inhibidor no selectivo de las ciclooxigenasas 1 y 2 y actúa bloqueando la síntesis de prostaglandinas e inhibe de manera reversible la agregación plaquetaria aumentando las hemorragias. Posee actividad analgésica y antiinflamatoria; se usa en el tratamiento de artritis reumatoide, osteoartritis, trastornos musculoesqueléticos, bursitis, tendinitis y sinovitis (8).

El Aloe vera L. "sábila" pertenece a las liliáceas, con más de doscientas especies descubiertas. Es oriunda de África Oriental y meridional, posee propiedades analgésicas, astringentes, antitóxicas, antifúngicas, antibacterianas, antivirales, antiinflamatorias, antioxidantes, antiulcerosas y es regeneradora de tejidos, pues posee polisacáridos y fenoles principales componentes activos del gel (9).

El gel, tiene principios activos, aminoácidos y hormonas que contribuyen a la regeneración celular y posee propiedades medicinales para sofocar ciertas dolencias tales como: acné, ulceras gastrointestinales, inflamaciones, pie de atleta, colitis, disentería, anemia, reumatismo, tuberculosis, cáncer y otros (10). 
En la investigación fitoquímica y farmacológica se realizan extracciones que sean reproducibles, cuantitativas, estables y eficientes para el efecto que se desea demostrar (11). Entre ellas, destacan las extracciones hidroalcohólicas (12) a base de éter, cloroformo y hexano. Existen pocos trabajos experimentales con modelos animales que comprueben la efectividad que puede tener el uso de la sàbila sobre las lesiones gástricas y si sus efectos son comparables o no con los procedimientos convencionales $(13,14)$. Con lo expuesto se planteó el objetivo de evaluar el efecto histoprotector de Aloe vera L. en ratas con daño gástrico inducido por indometacina.

\section{MATERIALES Y MÉTODOS}

La experiencia se realizó en la Universidad Toribio Rodrìguez de Mendoza, ubicado en la ciudad Chachapoyas, Perú. Se utilizaron 50 ratas hembras de la especie Rattus rattus var. albinus, de 4 meses de edad y 250 gramos de peso promedio; provenientes de la Facultad de Medicina de la Universidad Nacional de Trujillo. Los animales fueron acondicionados en el laboratorio durante 07 días como periodo de adaptación donde se mantuvieron en grupos de 10 ratas en jaulas metálicas incluyendo ciclos de luz-oscuridad; recibiendo agua y alimento ad libitum (30 gramos por animal de alimento comercial) con cambio diario de cama (viruta). En este periodo no se administró ninguna sustancia ni se realizó ningún tipo de procedimiento. El octavo día se consideró como día uno; luego se realizó el pesado de todos los animales y las observaciones necesarias que fueron asignadas en la ficha de observación (15) de cada espécimen. La inducción de úlceras en ratas se hizo con indometacina a una concentración de 20 $\mathrm{mg} / \mathrm{Kg}$ por vía hipodérmica según el sistema Sidney (16). Los animales fueron manipulados según las Normas de Ética de Experimentación Animal (17).

\section{Concentración óptima del gel de Aloe vera $\mathbf{L}$.}

Las hojas de A. vera L. (sábila) se colectaron de una planta de dos años de edad. . En el laboratorio las hojas fueron lavadas con agua y una solución de hipoclorito de sodio para evitar la contaminación bacteriana. Luego fueron colocadas en agua destilada en depósitos plásticos por 24 horas (con cambio de agua cada 12 horas) para eliminar las antraquinonas y toda impureza, previa eliminación de las espinas con un cuchillo. Se realizó cortes manuales a las hojas realizando un fileteado el gel con un cuchillo a partir de aproximadamente $2.5 \mathrm{~cm}$ desde la base de la hoja abarcando su extremo superior y las partes laterales (18), Se pesó $50 \mathrm{~g}$ de gel y se licuó con $150 \mathrm{~mL}$ de agua destilada conjuntamente con el exocarpio (19). Luego se hirvió por 10 minutos. Se concluye filtrando el producto y se colocó en un frasco de vidrio ámbar y estéril, para posteriormente refrigerarlo a $10^{\circ} \mathrm{C}(20)$.

\section{Cuantificación y dosificación del extracto de Aloe vera $\mathrm{L}$.}

En base a un trabajo piloto se determinó la dosis efectiva cincuenta $\left(D_{50}\right)$ del extracto de A. vera L. "sábila", el que fue diluido en agua destilada y administrado a los animales 
de experimentación según cada grupo de tratamiento correspondiente en dosis diarias por tres días. El trabajo piloto fue realizado con el $10 \%$ del número total de los animales de experimentación, a los cuáles se les administró vía hipodérmica diferentes concentraciones del extracto de A. vera $L$. donde se obtuvo la $\left(\mathrm{DE}_{50}\right)$ que fue de $50 \mathrm{mg} / \mathrm{Kg}$ de peso vivo.

\section{Diseño Experimental para evaluar el efecto histoprotector de A. vera $L$.}

Se utilizaron 5 agrupaciones de 10 ratas cada uno, distribuidos al azar asignados de una rotulación para su identificación.

\section{Agrupación 1: Control}

Agrupación 2: Se aplicó una dosis de indometacina.

Agrupación 3: Se ministró una dosis de $A$. vera L. y después de una hora se administró indometacina.

Agrupación 4: Se aplicó dos dosis de A.vera L. a sucesiones de 4 horas e indometacina a una hora de la primera dosis.

Agrupación 5: Se concedió tres dosis de A. vera L. a sucesiones de 4 horas e indometacina a una hora de la primera dosis.

Repeticiones. Se trabajó con 5 grupos de 10 animales cada uno.

Antes de los experimentos los animales estuvieron en ayuno por 24 horas, dejándolos únicamente con agua ad libitum. La indometacina se inyectó vía hipodérmica (diluída en agua destilada) en dosis de 15 $\mathrm{mg} / \mathrm{kg}$ p.v. Los procedimientos con A. vera $\mathrm{L}$. fueron aplicados con una cánula intragástrica y por vía oral en cantidad de $50 \mathrm{mg} / \mathrm{kg}$ p.v. (1.25\%). Al segundo día del experimento, se anestesiaron a los animales con pentobarbital sódico (halatal) vía intraperitoneal y se procedió a una laparotomía exploratoria Se aisló el antropíloro del estómago para la determinación de las lesiones. Los animales se sacrificaron por eutanasia, luego de tomada la muestra. Se analizaron los tejidos con observaciones macroscópicas y microscópicas. Luego las muestras fueron mantenidas en formol al $10 \%$ y trasladadas en frascos rotulados con su código individual, para el posterior análisis histopatológico.

\section{Evaluación del daño gástrico}

Para la evaluación de la injuria gástrica, la muestra resultante fue abierta linealmente por el lado de la curvatura mayor del epigastrio, separando la parte correspondiente del cuerpo y el antropíloro del estómago. Las lesiones gástricas se evaluaron utilizando la jerarquía de Marhuenda (20).

\section{Análisis estadístico}

En la valoración estadística los datos fueron procesados mediante la prueba T-Student, utilizándose el programa estadístico SSPS versión 21,0. Los datos fueron expresados como los promedios aritméticos \pm el error estándar del promedio aritmético, donde se aceptó un valor significativo de $p<0,05$. La comparación de grupos se realizó mediante la prueba no paramétrica de Kruskall Wallis. 
RESULTADOS Y DISCUSIÓN

Se propuso un modelo de gastritis erosiva inducida por indometacina, con un esquema similar al presenteestudio, en ratas de la especie Rattus rattus var. albinus, logrando lesiones en todos los animales, comprobándose que tales lesiones fueron inducidas en el duodeno y estómago respectivamente. En el presente estudio se pudo inducir las mismas lesiones en el lado antropilórico del estómago (es el sitio donde mayormente se origina la lesión ulcerosa en los mamíferos) y en aquellas áreas donde había presencia de vasos sanguíneos (21).

Tabla 1. Injurias gástricas macroscópicas (cuerpo y antropíloro) en $10 \mathrm{R}$. rattus var. albinus por agrupación provocadas por indometacina y procesadas con A. vera L.

\begin{tabular}{|c|c|c|c|c|c|c|}
\hline \multirow{2}{*}{ Exploración } & \multicolumn{5}{|c|}{ Agrupación de roedores con injuria } & \multirow{2}{*}{$\begin{array}{c}\text { Conjunto de } \\
\text { lesionados } \\
\text { a }\end{array}$} \\
\hline & $a^{b}$ & II & III & IV & $\mathbf{v}$ & \\
\hline Tumefacción de la cavidad gástrica & 0 & 5 & 3 & 2 & 2 & 7 \\
\hline Ampliación de la cavidad gástrica & 0 & 8 & 3 & 2 & 3 & 8 \\
\hline Mucosa dañada & 0 & 3 & 2 & 1 & 0 & 3 \\
\hline Hiperemia & 0 & 7 & 4 & 1 & 1 & 6 \\
\hline \multicolumn{7}{|l|}{ Secreción mucilaginosa } \\
\hline - ambarina & 0 & 5 & 2 & 0 & 0 & 2 \\
\hline - bermeja & 0 & 6 & 1 & 0 & 0 & 1 \\
\hline \multicolumn{7}{|l|}{ Ulceraciones } \\
\hline - $\quad$ singular y numerosas & 0 & 7 & 1 & 1 & 0 & 2 \\
\hline - longitudenales & 0 & 8 & 0 & 0 & 0 & 0 \\
\hline - carencia & 10 & 0 & 8 & 10 & 10 & 28 \\
\hline
\end{tabular}

${ }^{\text {aA }}$ un máximo de 30 (agrupaciones III, IV y V)

${ }^{\text {b} A g r u p a c i o ́ n ~ I: ~ C o n t r o l ; ~ I I: ~ I n d o m e t a c i n a ~(I) ; ~ I I I: ~ I ~ m a ́ s ~ u n a ~ d o s i s ~ d e ~ A l o e ~ v e r a ~ L ; ~ I V: ~ I ~ m a ́ s ~} 2$ dosis de A. vera L; V: I más 3 dosis de Aloe vera $\mathrm{L}$.

Los resultados microscópicos nos indican que las lesiones inflamatorias no están presentes en la mayoría de los animales, visualizándose la tumefacción de la cavidad gástrica e hiperemia como las cualidades más resaltantes en las agrupaciones III y V (Tabla 1 ). Es importante resaltar la ausencia de secreción mucilaginosa en las tres agrupaciones de animales tratados con Aloe vera L. así como la carencia de ulceraciones (Tabla 1 ). 
Tabla 2. Niveles de injuria macroscópica del estómago (cuerpo y antropíloro) en 10 R. rattus var. albinus por agrupación provocada por indometacina y procesadas con A. vera L. (20)

\begin{tabular}{ccccccc}
\hline $\begin{array}{c}\text { Nivel } \\
\text { c }\end{array}$ & $\begin{array}{c}\text { Agrupación I } \\
\text { b }\end{array}$ & Agrupación II & Agrupación III & Agrupación IV & $\begin{array}{c}\text { Agrupación } \\
\text { de } \\
\text { Agrupación V }\end{array}$ & $\begin{array}{c}\text { Lesionados } \\
\text { a }\end{array}$ \\
\hline A & 10 & 1 & 3 & 3 & 4 & 10 \\
B & 0 & 1 & 4 & 1 & 1 & 6 \\
C & 0 & 0 & 2 & 2 & 1 & 5 \\
D & 0 & 0 & 3 & 1 & 1 & 5 \\
E & 0 & $7(2)$ & 1 & 2 & 1 & 4 \\
\hline
\end{tabular}

a A un máximo de 30 (agrupaciones III, IV y V)

${ }^{\text {b} A g r u p a c i o ́ n ~ I: ~ c o n t r o l ; ~ I I: ~ i n d o m e t a c i n a ; ~ I I I: ~ i n d o m e t a c i n a ~ m a ́ s ~ u n a ~ d o s i s ~ d e ~ A l o e ~ v e r a ~ L ; ~ I V: ~ i n d o m e t a c i n a ~ m a ́ s ~ d o s ~ d o s i s ~ d e ~ A . ~ v e r a ~ L ; ~ V: ~ i n d o m e t a c i n a ~}$ más tres dosis de A. vera L.

'Nivel A (normal): Mucosa y serosa saludable

Nivel B: (leve): hiperemia y/o hemorragia con pequeñas áreas sanguinolentas (petequias)

Nivel C: (moderado): mucosa escoriada o ulcerada

Nivel D: (severo): mucosa escoriada o ulcerada con injurias y sangrado.

Nivel E: (muy severo): numerosas llagas o úlceras

Respecto a las lesiones macroscópicas, según la jerarquía de Marhuenda (20), se observó la escaza presencia de injurias numerosas o ulceras (nivel E) (Tabla 2) excepto la agrupacíon II; sin embargo se percibió una ligera mucosa erosionada con sangrado de nivel D (Tabla 2). Tal como se muestra en la figura 4, las células epiteliales de la mucosa sana están migrando hacia la zona de injuria, proliferando en ella, para restaurar la lesión de la mucosa.

Tabla 2. Niveles de injuria microscópica del estómago (cuerpo y antropíloro) en10 Rattus rattus var. albinus por agrupación provocada por indometacina y procesadas con A. vera L. (20)

\begin{tabular}{ccccccc}
\hline $\begin{array}{c}\text { Nivel } \\
\text { c }\end{array}$ & $\begin{array}{c}\text { Agrupación I } \\
\text { b }\end{array}$ & Agrupación II & Agrupación III & Agrupación IV & $\begin{array}{c}\text { Ratas estimadas } \\
\text { Agrupación V Vación } \\
\text { de } \\
\text { Lesionados } \\
\text { a }\end{array}$ \\
\hline Sin lesión & 10 & 0 & 2 & 4 & 4 & 10 \\
Ligero & 0 & 2 & 3 & 3 & 0 & 6 \\
Regular & 0 & 1 & 1 & 2 & 5 & 8 \\
Grave & 0 & 7 & 3 & 2 & 1 & 6 \\
\hline
\end{tabular}

${ }^{a}$ A un máximo de 30 (agrupaciones III, IV y V)

b Agrupación I: control; II: indometacina; III: indometacina más una dosis de Aloe vera L.; IV: indometacina más dos dosis de Aloe vera L.; V: indometacina más tres dosis de A. vera L.

'Nivel Ligero: Involucra ausencia de daño tisular, exiguas pruebas de deterioro de vasos sanguíneos (hiperemia e hinchazon) y poca secreción. Nivel Regular: El efecto siempre involucra un fragmento tisular y descubrimientos evidentes de consecuencias inflamatorias al daño, comprobada por la aglomeración de glóbulos blancos y acontecimientos en los vasos sanguíneos.

Nivel Grave: Abarca la ampliación del efecto moderado en la cual se percibe un intenso deterioro tisular con alta prolongación y mucha secreción y/o exudación. 
Respecto a la Tabla 3, lo hallado y por lo menos dos de ellos no presentaron microscópicamente según la jerarquía de Marhuenda (20) existen entre tres y dos animales por grupo que presentaron lesiones graves, lo cual representa más del $50 \%$ del total de animales tratados con Aloe vera L.; algún tipo de injuria. Debe recalcarse también que los animales de los agrupaciones III y IV (una dosis, dos dosis) presentaron más lesiones ligeras que la agrupación $\mathrm{V}$ que recibió tres dosis de Aloe vera $\mathrm{L}$.

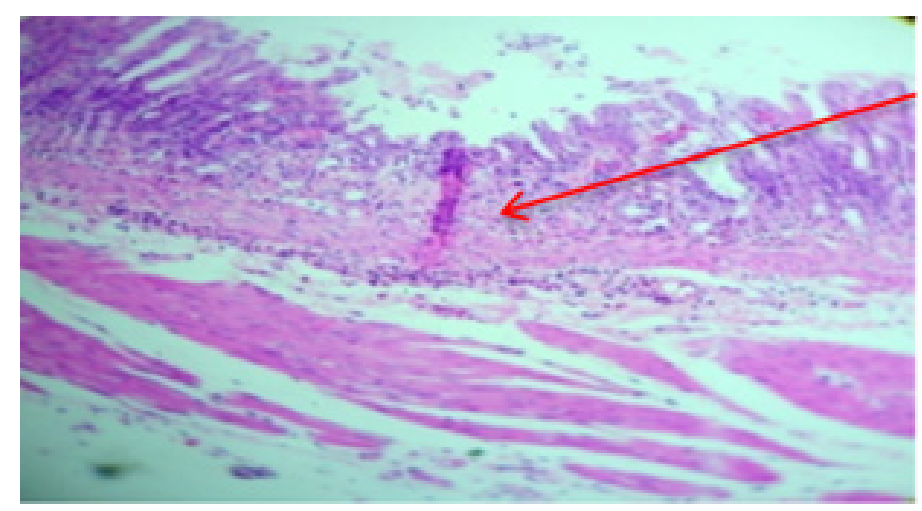

\section{MUCOSA EN \\ PROCESO DE \\ RESTAURACIÓN}

Figura 1. Zona antropilórica del estómago (agrupación 2) donde se observa una mucosa necrosada con atrofia glándular.

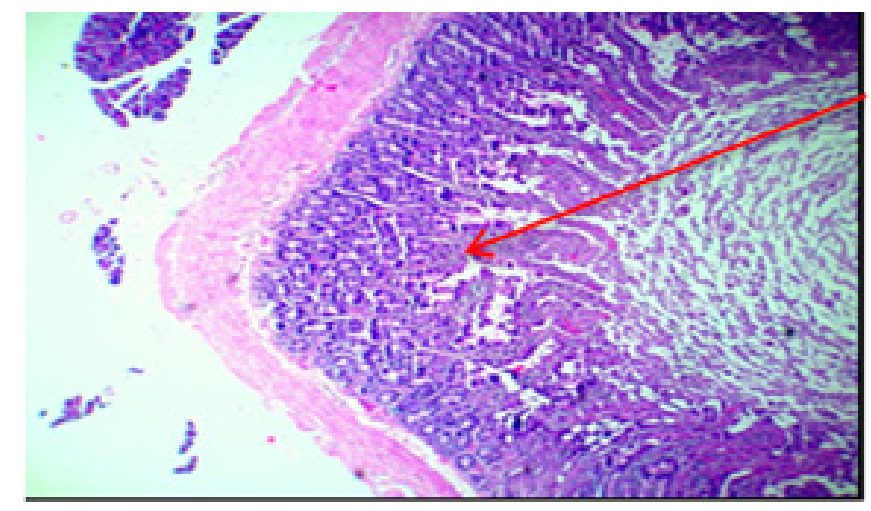

\section{MUCOSA \\ EROSIONADACON}

CORIÓN ALTERADO

Figura 2. Zona corporal del estómago (agrupación 3) donde se observa la erosión de la mucosa y el corión alterado.

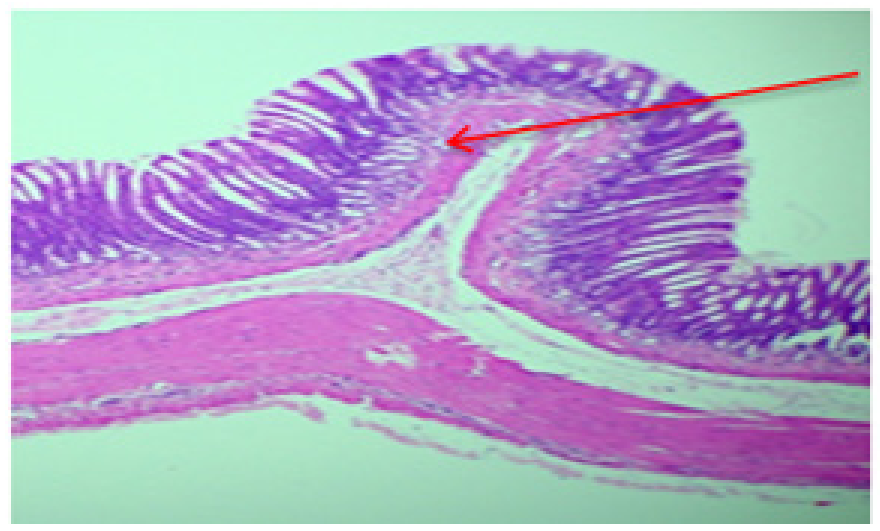

\section{MUCOSA GÁSTRICA}

REGENERADA

Figura 3. Zona antropilórica del estómago (agrupación 4) donde se observa la mucosa regenerada. 


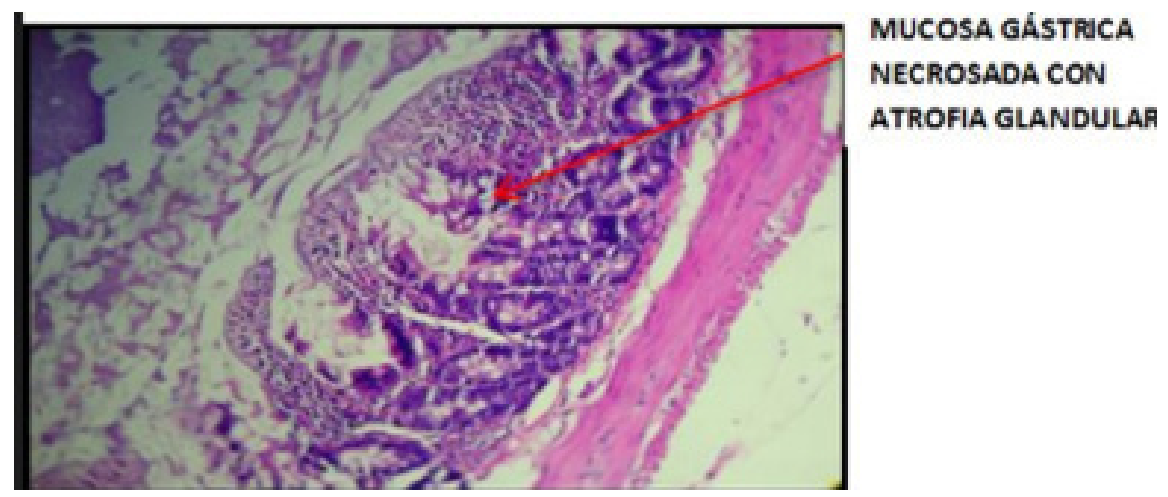

Figura 4. Zona del cuerpo del estómago (agrupación 5) observándose células epiteliales de mucosa sana migrando hacia la zona de injuria para restaurar la lesión de la mucosa.

\section{Discusión}

Conociendo el poder gastrolesivo de la indometacina (22) (Figura 1) en animales de experimentación, se aplicó periódicamente vía hipodérmica cada 4 horas a diferentes dosis de extracto de A. vera L. "sábila". Se comprobó el efecto bioprotector de A. vera L. "sábila" en $R$. rattus var. albinus con daño gástrico inducido por indometacina; pues el extracto de "sábila" posee los 11 aminoácidos esenciales que fueron puestos a disposición de la maquinaria reparadora de las células mitóticas de la mucosa de la parte antropilórica del estómago evidenciándose (Tabla 1) esto en las agrupaciones 3,4 y 5 donde no existió exudado mucoso (Figura 2) y se observó escasa presencia ulcerosa en la mayoría de animales tratados con A. vera L. (23).

Sabiendo que la formación ulcerosa se debe a la acción de la indometacina $(24,25)$ la cual suprime a las enzimas llamadas ciclooxigenasas (Cox uno y Cox dos) y a las prostaglandinas I y E2 (PGI y $P G E)$, estas biomoléculas confieren a la mucosa una función protectora estimulando la formación de mucus y bicarbonato para contrarrestar el efecto del ácido clorhídrico. Es aquí donde la sábila presenta sustancias estimuladoras para la formación de las ciclooxigenasas y prostaglandinas logrando proteger a la mucosa intestinal de la actividad del ácido clorhídrico. En la Tabla 1 se puede apreciar que de 30 animales inducidos con daño gástrico por la indometacina, 28 de ellos no presentaron ulceras gástricas; esto se atribuiría al extracto vegetal cuya propiedad fue activar, fortalecer y hacer más eficiente el sistema histoprotector de la mucosa erosionada asegurando una renovación conjunta de tejidos ante la injuria ocasionada por la indometacina. También nuestros resultados son similares a lo encontrado por Celestino y López (26) quienes indujeron ulceras gástricas en ratas al usar un extracto etanólico de Aloe vera L., los animales presentaron signos de mejoría por el efecto gastroprotector inducido con sábila.

El $90 \%$ de las ratas de las agrupaciones 4 y 5 a las que solo se les administró dos y tres dosis de $A$. vera $L$. se logró reducir el daño ulceroso en la mucosa gástrica encontrándose 
estadísticamente diferencias significativas $(p<0,05)$ con la agrupación 2 que solo recibió indometacina (hallándose en ellos más del $80 \%$ de animales con daño de la mucosa gástrica). Estos resultados concuerdan con lo hallado por Salvatierra (27) quien un estudio similar al nuestro induciendo lesiones gástricas en ratas con indometacina, observó que la introducción de una dosis de sábila entre el grupo preventivo y el grupo curativo no se halló diferencias significativas. Se conjetura que la administración de sábila en el momento oportuno y exacto del experimento produjo a nivel de la mucosa la estimulación de ciertas prostaglandinas que favorecen y estimulan la producción de moco con bicarbonato de sodio produjo una intensa mitosis de las células epiteliales como se demuestra en la figura 3 y 4 mostrando una mucosa gástrica protegida y con menor presentación de lesiones erosivas y/o inflamatorias. Se demostró que la aplicación de gel de sábila sobre heridas pos-endodoncia en ratas, estas mostraron mejoría (28), o sea que a nivel bucal la cualidad desinflamatoria de la sábila está demostrada.

Discrepamos con Gutiérrez (29) con lo encontrado en su estudio quien utilizando indometacina como agente inductor gastrolesivo descubrió un moderado porcentaje $(60 \%)$ de animales con petequias en el tratamiento donde solo se usó indometacina; mientras en nuestro análisis (Tabla 2), las agrupaciones 4 y 5 que recibieron dos y tres dosis de "sábila" se encontró un $60 \%$ y $90 \%$ que no exhibió petequias. El mencionado autor usando solo indometacina en la agrupación control halló que el $40 \%$ de los animales presentó lesiones moderadas mientras que en nuestra investigación tal agrupación control presentó solo el $20 \%$ de lesiones ligeras o moderadas quizá la dosis utilizada en nuestro estudio $(20 \mathrm{mg} / \mathrm{Kg})$ influyó en los resultados y esto resalta en los resultados.

En la Tabla 3 respecto a las lesiones macroscópicas según la escala de Marhuenda se apreció en todos los tratamientos la ausencia de ulceraciones mayores de $10 \mathrm{~mm}$. De las agrupaciones 3,4 y 5 , es decir, de los 30 animales tratados (100\%) el $86,7 \%$ no presentó ulceraciones menores de $10 \mathrm{~mm}$. Armonizamos con el estudio realizado por Gallardo y Ramos (30) quienes encontraron un $78 \%$ de eficiencia histoprotectora de $A$. vera $L$. en un modelo experimental en ratas. La presencia de estas erosiones ulcerosas podría deberse a la acción de los radicales libres producto de la presencia leucocitaria que tiene como objetivo la erosión y la necrosis de la mucosa que son componentes de la pared gástrica; sin embargo, ciertos agentes antioxidantes coma las vitaminas $A$ y $C$ presentes en $A$. vera L. contrarrestaron posiblemente el efecto de los radicales libres evitándose así el deterioro masivo de tejidos del estómago y, por ende, la formación ulcerosa. Otros autores (31, 32) encontraron que polisacáridos aislados de A. vera L. denominados GAPS-1 (gel) y SAPS-1 (exocarpio) están compuestos de manosa:glucosa:galactosa los cuales tienen una fuerte acción antioxidante eliminando los iones superóxido y hidróxilo así como la peroxidación lipídica. Además, debemos 
destacar los flavonoides que contiene $A$. vera $\mathrm{L}$. que complementaron la actividad antioxidante en el tejido dañado. Los datos obtenidos sugieren que la etapa de madurez de la sábila tiene un papel importante en la composición y en la actividad antioxidante de la misma; los resultados de este estudio corroboran que el tiempo de madurez de la sábila fue determinante.

En Tabla 3, los hallazgos microscópicos muestran que entre tres y dos animales (agrupaciones 3, 4 y 5) presentaron lesiones graves $y / 0$ severas, lo cual demuestra que el $80 \%$ del total de animales (30 animales igual a 100\%) no presentaron estos efectos injuriosos deduciéndose que por efecto de las diferentes dosis de A. vera $L$. administrados a los animales, estos tejidos dañados lograron regenerarse como se aprecia en las figuras 3 y 4; habiendo un promedio de 3 animales que no presentó ninguna lesión (estado saludable). Este estudio coincide con los resultados obtenidos por Gonzales et al. (33) que en un estudio encontró que el efecto de un extracto de papa, uña de gato y muña en ratas fue del $96,2 \%$ de efectividad antiulcerosa. Superando inclusive al efecto antiulceroso de la ranitidina (usado como grupo comparativo) que mostro un efecto de 50,01\%.

Conociendo que el efecto antiinflamatorio, analgésico y antifebril son comunes a los AINES y que, a su vez, estos presentan sus mayores efectos secundarios y gastrolesívos a nivel de la mucosa gástrica e intestinal; entonces estudio planteó estudiar el mismo principio para indagar el efecto que pudiera tener en el tratamiento experimental de ulceras gástricas inducidas por indometacina en ratas, habiendo comprobado lo que nos propusimos al encontrar que la dosis de A. vera L. de $50 \mathrm{mg} /$ $\mathrm{kg}$ muestra un efecto regenerador al disminuir las lesiones gástricas. Esto probablemente se deba a los principios activos de las quinonas que posee $A$. vera $L$.

CONCLUSIONES

Se comprobó el alto efecto histoprotector de A. Vera L. "sábila" en ratas con daño gástrico por indometacina al aplicar una, dos y tres dosis de sábila por cada tratamiento.

El extracto de Aloe vera L. aplicado en una, dos y tres dosis a Rattus rattus var. albinus con daño gástrico posee un notable efecto histoprotector al presentar en su estómago una mucosa sana con ausencia de úlceras, escaza hiperemia y sin necrosis.

Las tres dosis del extracto de Aloe vera $\mathrm{L}$. "sábila" aplicadas a los animales disminuyó en un $\mathbf{8 6 , 7 \%}$ las lesiones ulcerosas inducidas por indometacina.

\section{REFERENCIAS}

1. Harrison T. Principios de Medicina Interna. McGraw-Hill S.A. México. 2016.

2. Viruete $S$. Manual de conocimientos básicos de Farmacología. Universidad de Guadalajara. Mexico. 2015.

Katzung B, \& Trevor, A. Farmacología Básica y Clínica. Mc Graw Hill S.A. México. 2016.

3. Gonzales L. Llanos J. \& Chotón M. Efecto antiulceroso de Aloe vera L. "sábila", miel de Apis mellifera L. "miel de abeja” y cañazo en Rattus rattus var. albinus con lesiones gástricas inducidas por etanol. Conocimiento para el Desarrollo. 2014; 25-31. 
4. Bussmann RD. Sharon. Plantas medicinales de los Andes y la Amazonía. Edit. Jardín Botánico de Misouri, Trujillo. Perú. 2015.

5. Coria S. Efecto gastroprotector del extracto hidroalcohólico de las hojas frescas de Spinacia oleraceae L. "espinaca" en ratas. Tesis de Licenciatura. Universidad Norbert Wiener. Lima. Perú. 2019.

6. Inocente T. Efecto gastroprotector del extracto hidroalcoholico de las hojas de Mutisia acuminata r. \& p. "chinchemano" en lesiones gástricas inducidas por naproxeno sódico. Tesis de Licenciatura. Universidad Norbert Wiener. Lima. Perú. 2017.

7. Chirri W. Estudio comparativo de la acción gastroprotectora del Plantago major y el omeprazol sobre la gastritis inducida por la administración de ketorolaco. Revista Peruana de Medicina. 2017; 85-91.

8. Alfaro A. Efecto gastroprotector del extracto hidroalcohólico de las hojas de Juglans neotropica Diels (Nogal) en Rattus rattus var. Albinus con úlceras gástricas inducidas por indometacina. Tesis de Licenciatura. Universidad Católica Los Ángeles de Chimbote. Trujillo. Perú. 2018.

9. Gonzales L, \& Díaz C. Efecto citoreparador de Aloe vera L. "sábila" en tejidos embrionarios de Allium cepa L. "cebolla" con daño cromosómico inducido por amoxicilina. Revista Acc Cietna. 2016; 1-10.

10. Lezaeta M. La medicina natural al alcance de todos. Buenos Aires. Argentina. 2016.

11. Rang $H$, Flower $R$, Ritter $M$, \& Anderson G. Farmacología. Studentconsult. Editorial Elsevier. 2016.

12. Román $\mathrm{H}$, y Zedano D. Efecto antiulceroso del extracto hidroalcóholico de las hojas de Mutisia acuminata en Rattus novergicus Holtman. Tesis de licenciatura. Universidad María Auxiliadora. Lima-Perú, 2019.

13. Muñoz L. Estudio farmacognóstico y cuantificación de flavonoides totales de las hojas de Bixa Orellana (Achiote) provenientes del distrito de Usquil, provincia de Otuzco, región la libertad. Tesis de licenciatura. Universidad Nacional de Trujillo. Perú. 2015.

14. Mamani Y. Evaluación del efecto antiulceroso y toxicidad aguda del extracto hidroalcohólico al $70 \%$ de la corteza de Triumfetta bogotensis (rata- rata) en animales de experimentación. Tesis de Licenciatura. Universidad Nacional Mayor de San Antonio Abad del Cuzco. Perú. 2017.

15. Beltrán R. Metodología de la Investigación Científica. CONCYTEC. Lima. Perú. 2015.

16. León M. Efecto antiulceroso del extracto hidroalcohólico de Plantago lanceolata (llantén menor) sobre la úlcera gástrica inducida en ratas. Tesis de Licenciatura. Universidad Nacional Mayor de San Marcos. Lima. Perú. 2016.

17. Pardo C. Animal experimentation ethics. Contemporary legal and ethical rules. Cuad Bioét. 2005; 393-417.

18. Domínguez R, Arzate J. Chanona J, Welti J, Alvarado G, Calderón V, \& Gutiérrez G. El gel de Aloe vera: estructura, composición química procesamiento, actividad biológica e importancia en la industria farmacéutica y alimentaria. Revista Mexicana de Ingeniería Química. 2012; 23-43.

19. Herencia V, Rivera I, Correa L, \& De la Cruz J. Efecto gastroprotector de un nutracéutico compuesto por Ocimum micranthum Willd (albahaca silvestre) frente a ulceras gástricas inducidas por etanol en ratas. Revista Peruana de Medicina. 2018; 91-97.

20. Marhuenda, R, \& Bravo D. Manual de Farmacoterapia. Elsevier. Madrid. 2005.

21. Torres D. Efecto protector del extracto hidroalcoholico de hojas de Aloe vera L. Y Argemone subfusiformis Own. en lesiones gástricas inducidas con etanol en Mus musculus BALB/C". Tesis de Licenciatura. Universidad Nacional de Trujillo. Perú. 2014.

22. Sánchez A. Efecto gastroprotector del extracto alcohólico de las hojas de Plantago Major (LLantén) frente a ranitidina en Rattus rattus var. Albinus con úlcera gástrica inducida 
por indometacina. Tesis de Licenciatura. Universidad Católica Los Ángeles. Chimbote. Perú. 2019.

23. Gonzales L, Chotón M, \& Chico J. Un extracto combinado de Echinopsis pachanoi L. "San Pedro" y Aloe vera L. "sábila" disminuye las lesiones por gastritis hemorrágica en Rattus rattus var. albinus. Manglar. 2020; 147-152.

24. Flores R, \& Flores Y. Efecto gastroprotector y curativo del consumo de harina de vaina de tara (Caesalpinia spinosa) en animales de experimentación inducidos a Gastritis. Tesis de Licenciatura. Universidad Nacional de San Agustín. Arequipa. Perú. 2017.

25. Leyva J. Evaluación de la toxicidad gástrica con el tratamiento de indometacina, diclofenaco y omeprazol en ratas. Universidad Autónoma de Guerrero. Thamati Sabiduría. México. 2016; 1-12.

26. Celestino K, \& López J. Efecto cicatrizante de un gel a base del extracto etanólico de la hojas de ortiga (Urtica urens L.) y extracto etanólico del mucílago de la sábila (Aloe vera L. Burn.) en ratas albinas. Tesis de Licenciatura. Universidad Inca Garcilaso de la Vega. Lima. Perú. 2018.

27. Salvatierra A. Efecto protector del gel de Aloe vera en lesiones gástricas inducidas con indometacina en Rattus rattus var. Albino. Tesis de Licenciatura. Universidad Cesar Vallejo. Trujillo. Perú. 2015.

28. Morales T. Efectividad cicatrizante del gel de propóleos y gel de Aloe vera sobre heridas post exodoncia simple en Rattus rattus. Tesis de Licenciatura. Universidad de los Ángeles. Chimbote. Perú. 2018.

29. Gutiérrez, W. Evaluación de los compuestos fenólicos del extracto de las hojas de muña (Minthostachys spicata) en el queso tipo paria. Tesis para Licenciatura. Universidad Nacional del Altiplano. Puno. Perú. 2017.

30. Gallardo E, \& Ramos J. Efecto gastroprotector del extracto acuoso de Aloe vera "sábila" vs extracto hidroalcohólico de Piper aduncum "matico" en Rattus rattus var. albinus con úlceras gástricas inducidas por indometacina. Tesis de Licenciatura. Universidad Particular Antonio Urrelo. Cajamarca. Perú. 2018.

31. Toso F, Lara M, Mondino M, Marron Y, \& Pombar A. Actividad gastroprotectora de Equisetum giganteum L. n.v. cola de caballo y Cortaderia selloana (Schult \& Schult. f.) n.v. cortadera en ratones sometidos a estrés e indometacina. Ciencia Veterinaria. 2019; 43-52.

32. Chun-hui L, Chang-hai, W, Zhi-liang X, \& Yi W. Isolation, chemical characterization and antioxidant activities of two polysaccharides from the gel and the skin of Aloe barbadensis Miller irrigated with sea water. Process Biochemistry. 2007; 961-970.

33. Gonzales L, Chico J, \& Chotón M. Efecto citoprotector del extracto mixto de Solanum tuberosum L. "papa", Minthostachys mollis L. "muña" y Uncaria tomentosa L. "uña de gato" en las lesiones ulcerosas de ratas inducidas por etanol. Rebiol. 2020; 177-187. 